\title{
PERFORMANCE OF ROUGHNESS CORRECTION MODELS FOR RETRIEVAL OF SEA SURFACE SALINITY FROM AIR- AND SATELLITE-BORNE L-BAND RADIOMETERS
}

\author{
Derek Burrage ${ }^{\prime}$, Joel Wesson ${ }^{\prime}$, Paul Hwang ${ }^{2}$, and David Wang ${ }^{1}$ \\ Naval Research Laboratory, \\ ${ }^{1}$ Oceanography Division, Code 7300, Stennis Space Center, MS, USA \\ ${ }^{2}$ Remote Sensing Division, Code 7200, DC, USA \\ Emails: burrage,wesson,dwang@nrlssc.navy.mil, phwang@ccs.nrl.navy.mil
}

\begin{abstract}
The recent and imminent launch of the SMOS and Aquarius satellites carrying microwave L-band radiometers provides an opportunity to map Sea Surface Salinity (SSS) globally with an expected error $<0.2$ psu. However, the accuracy of retrieved SSS depends critically on brightness temperature (Tb) corrections for sea surface roughness (SSR) effects. This paper assesses the performance of representative roughness correction models when compared with published data, and applied to recently-acquired airborne Lband radiometer data. One type of model currently being used to process SMOS data combines a wind-driven gravity wave spectrum that describes SSR, with an electromagnetic (EM) model that determines microwave emissivity, to predict the $\mathrm{Tb}$ roughness increment relative to the flat sea response. We find that selection of both the spectral and emissivity models strongly influences the resulting $(\sim 1 \mathrm{~K})$ $\mathrm{Tb}$ errors. We conclude that more accurate modeling of short wavelength spectral components and their EM influence is needed, to reduce these errors to acceptable levels.
\end{abstract}

Index Terms - L-band Radiometer, Sea Surface Salinity, Emissivity, Surface Roughness, Wave Spectra

\section{INTRODUCTION}

The launches of ESA's Soil Moisture and Ocean Salinity, SMOS, L-band microwave satellite [8] in Nov., 2009, and NASA's Aquarius satellite [19] in late 2010, promise monthly global maps of Sea Surface Salinity (SSS) over the deep ocean with $0.2 \mathrm{psu}$ precision at $100 \mathrm{~km}$ resolution. However, SSS retrieval accuracy depends critically on corrections for SSR effects. These effects change the Lband $(\sim 21 \mathrm{~cm}$ wavelength) sea surface brightness temperature, $\mathrm{Tb}$, predicted by 'flat sea' emissivity models [17], as much as do open ocean surface salinity variations. Thus, they require careful correction. This paper describes the evaluation of roughness correction models designed for retrieving SSS from L-band microwave radiometer $\mathrm{Tb}$ measurements over rough seas. The paper first describes the airborne instrumentation, and the wave spectrum and emissivity models used. Then results from several models are presented and compared using published data and recent NRL Salinity, Temperature and Roughness Remote Scanner, STARRS, airborne salinity mapper campaign measurements. It is shown that use of Hwang's recently published wave spectrum [12] to drive the SSA/SPM [15] [16] [22] [25] emissivity model can eliminate the need for an overall factor of two increase in spectral intensity used in previous roughness correction models.

\section{METHODS}

\subsection{Airborne Instrumentation}

STARRS comprises L and C-band microwave radiometers, an infrared (IR) radiometer, integrated GPS receiver, and gyro. The combined measurements are used to retrieve sea surface salinity (SSS), temperature (SST) and roughness. The L-band radiometer, used primarily to retrieve SSS, is a multi-beam system sensing microwave thermal emission from the sea surface within a $24 \mathrm{MHz}$ wide protected band, centered at a frequency of $1.4 \mathrm{GHz}$. The 6 antenna beams, with 15 deg half power beam width, point downward and to either side of the aircraft at incidence angles of $+/-7,22$ and 38 degrees. The nadir-viewing IR radiometer senses SST from thermal emission in the 8-14 and 9.6-11.5 micron bands. For typical aircraft speeds of $80 \mathrm{~m} / \mathrm{s}$ and altitudes of $2600 \mathrm{~m}$, the beam geometry yields a $5.2 \mathrm{~km}$ swath width. See [1] and [20] for other instrument and processing details.

\subsection{Roughness Emissivity Models}

Various emissivity models are presently available to correct for the adverse effects of roughness-enhanced emission on microwave SSS retrievals. These include rigorous [21], asymptotic [16] [22] [26] and empirical model types [2] [3] [9]. Three models of the last two types are implemented in 

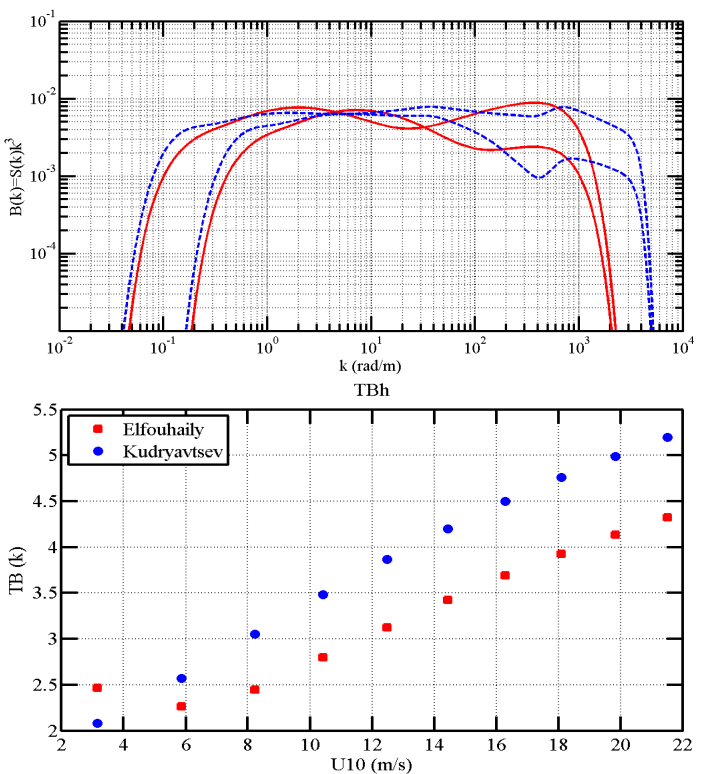

Fig 1. SPM/SSA calculations from different wind-wave Spectra. (Top) Curvature spectra $B(k)$ of Elfouhaily et al. [5] and Kudryavtsev et al. [13] versus wavenumber $\mathrm{k}(\mathrm{rad} / \mathrm{m})$. (Bottom) Predicted $T b(\mathrm{~K})$ versus wind speed $U(\mathrm{~m} / \mathrm{s})$ at $10 \mathrm{~m}$ from SSA/SPM for Inc.Ang. 37 deg., Ts $298 \mathrm{~K}, S 35 \mathrm{psu}$, Inv.wave age $=0.84$.

the SMOS Level 2 processor. The asymptotic models are driven by auxiliary wind data and based on a specified wind-wave spectrum. Their accuracy is strongly influenced by the choice of spectrum. The empirical models are driven directly by auxiliary wind or sea state data, and calibrated using in situ observations. They take advantage of SMOS's multi-angle view capability [8]. In contrast, Aquarius will model roughness empirically using radar cross sections observed by an on-board L-band scatterometer [19]. The variety of models adopted, even for the single mission, SMOS, is a reflection of recent issues and innovations in this field, and of the dominant role of sea surface roughness corrections in the L-band radiometer error budgets.

\subsection{Wave Spectral Models}

The asymptotic emissivity models are combined with a wind-driven SSR spectrum to form a complete roughness correction model. From the perspective of determining microwave emissivity, the gravity wave spectra remain poorly defined. The difficulties of specifying an optimal SSR spectrum is highlighted in the comparison between field measurements and analytical computations. For example, Yueh [26] selects the Durden and Vesecky spectrum [6] for his two-scale model, but must double the roughness spectral densities to obtain reasonable agreement with field measurements. Camps et al. [2004] used the [6] and Elfouhaily et al. [7] spectral models, also doubled, to make the calculated rate of change of brightness temperature with wind speed agree roughly with their

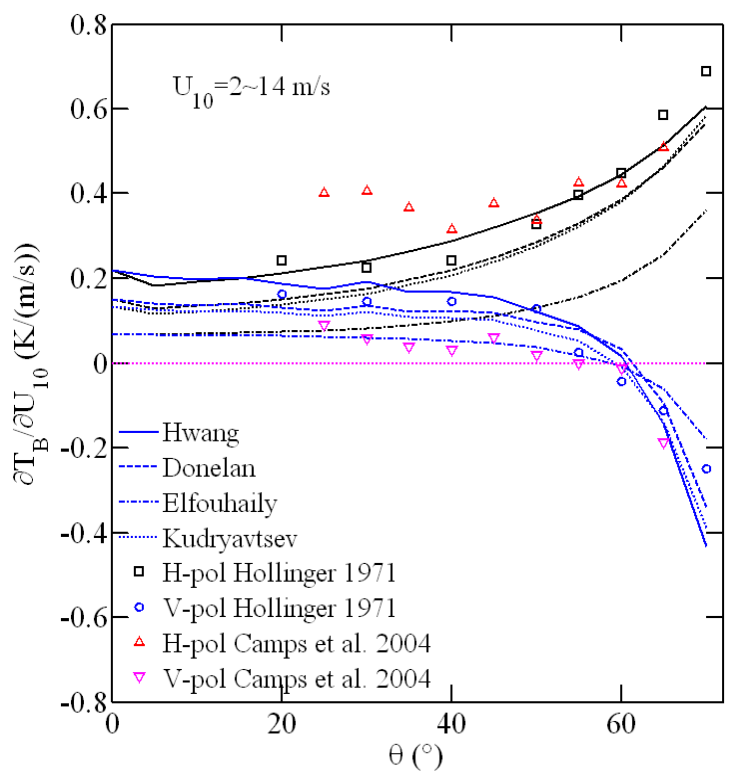

Fig 2. Average rate of change of $T b$ with respect to wind speed, $\partial T b / \partial U 10(\mathrm{~K} / \mathrm{m} / \mathrm{s})$ versus incid. angle at $1.41 \mathrm{GHz}$ calculated using four different wind-wave spectra for wind speed range 2 to $14 \mathrm{~m} / \mathrm{s}$. Hollinger [10] and Camps et al. [3] field data are superimposed.

measurements. While [6] produces mean square slope (MSS) similar to the classical sun glitter measurements of Cox and Munk [4], Yueh [26] argued that other well known spectral models produce integrated total MSS about twice the magnitude of the CM data, which could have underestimated surface slopes that were very steep, but infrequent [23]. Similar difficulty in specifying the SSR spectrum is also encountered in active radar scattering computations [13] [14].

\section{RESULTS}

\subsection{Roughness Correction Model Assessment}

To investigate the importance of model choice in computing roughness corrections for SSS retrieval, we compared results from the Two-Scale Model (TSM) of Yueh [26] and Reul's (pers. comm.) code for the SPM/SSA asymptotic model [16][22], both of which are implemented in the SMOS L2 processor. These models also employ different wind-wave spectra to describe sea roughness. SPM/SSA is driven by the Kudryavtsev, et al. spectrum [18] and TSM by the Durden-Vesecky spectrum [6] (multiplied by two, as discussed above), with Gaussian-distributed long wave slopes [26]. A significant difference in the Tb's predicted by TSM versus the SPM/SSA model appeared, particularly for $\mathrm{H}-\mathrm{Pol}$. At $50 \mathrm{deg}$. incidence angle, TSM predicts $\sim 2 \mathrm{~K}$ lower $\mathrm{Tb}$ influence than SPM/SSA ( 4 psu SSS error!). Such under-prediction by TSM has previously been reported by other investigators. 

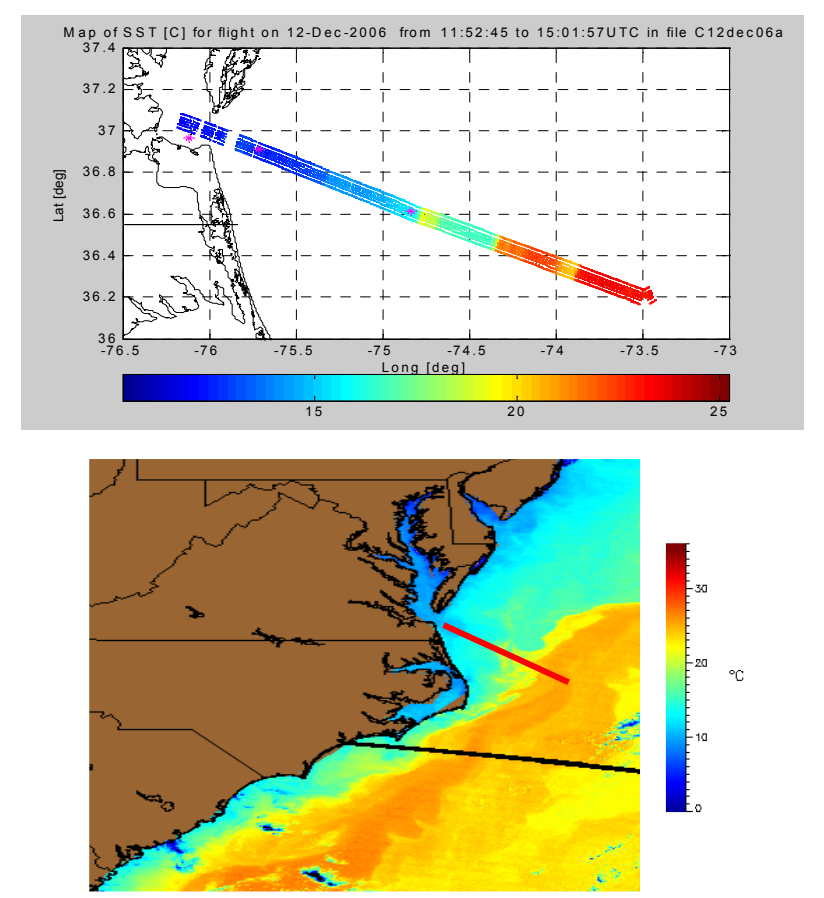

Fig 3. (Top) STARRS SST transect from Chesapeake Bay entrance to mid Gulf Stream. (Bottom) MODIS satellite SST image showing transect crossing Gulf Stream (Red line is STARRS transact, black line is a satellite data dropout).

\subsection{Effect of Spectral Model}

To illustrate the effect of input spectrum choice for a single asymptotic emissivity model, we show two surface curvature spectra (Fig. 1) and resulting predictions of Lband H-Pol Tb's, derived using Reul's implementation of the SPM/SSA model [22]. The input wave spectra were those of Kudryavtsev et al. (K) [18] and Elfouhaily et al. (E) [7]. The resulting Tb predictions differ by $\sim 1 \mathrm{~K}$ for an SSS error of about 2 psu under typical temperate conditions (VPol errors, not shown, were of similar magnitude). Comparison of $\mathrm{Tb}$ wind sensitivity, which results from using $\mathrm{E}$ and $\mathrm{K}$ along with the Donelan (D) [5] and Hwang (H) [12] spectrum to drive SPM/SSA (Fig. 2), shows that $\mathrm{H}$ performs best for $\mathrm{H}-\mathrm{Pol} \mathrm{Tb}$ and is competitive with $\mathrm{K}$ for $\mathrm{V}$ Pol, considering field data spread. Thus $\mathrm{H}$ provides the best overall performance, while both models produce wind sensitivities appreciably larger than those predicted using the E spectrum.

\subsection{Application to Airborne Measurements}

A STARRS transect crossing the continental shelf and western half of the Gulf Stream conducted during NRL's VIRGO experimental campaign in Dec., 2006 shows SST's obtained from the STARRS and MODIS IR radiometers and Gulf Stream location (Fig. 3), and the corresponding
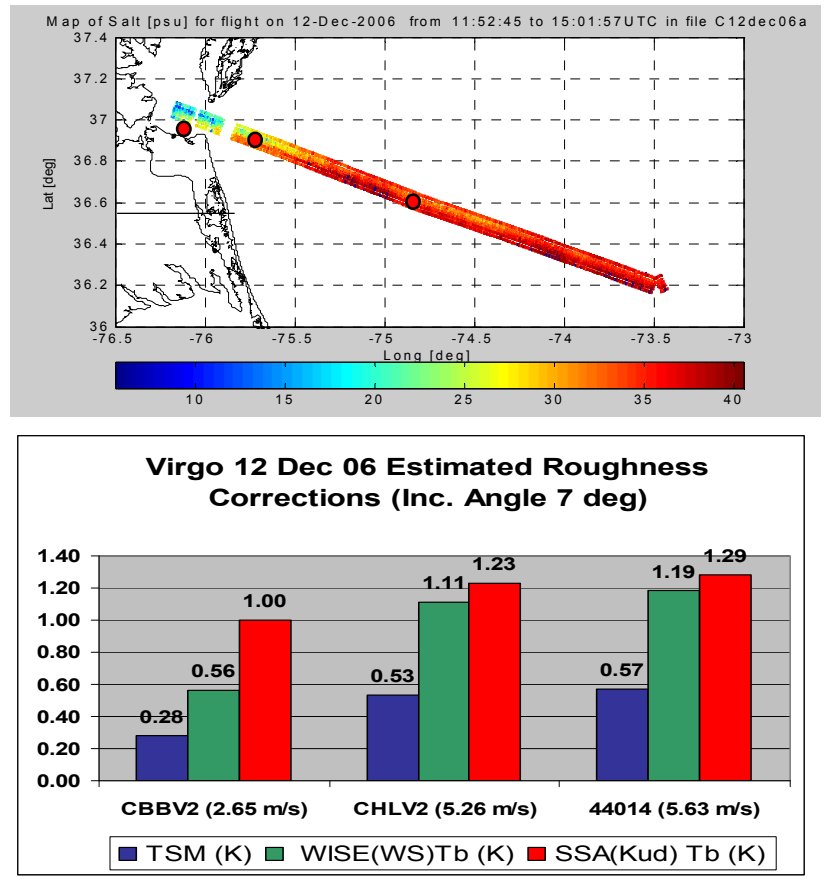

Fig 4. (Top) STARRS SSS transect passing NDBC buoys (red dots, W to E CBBV2, CHLV2 and 44014). (Bottom) Bar chart showing L-band $T b[\mathrm{~K}]$ corrections from TSM (blue), WS (green), and SPM/SSA (red) for the buoy locations, and observed wind speeds [m/s] (see labels).

STARRS SSS transect (Fig. 4). The bar chart shows the VPol Tb corrections (Delta Tb) computed from TSM [26], the empirical WISE emissivity model [2] (WS), and SPM/SSA (with input $\mathrm{K}$ spectrum), at locations near NOAA data buoys. The wind speeds observed by the buoys during the flight are also shown. The corresponding SSS correction in this temperate region, in psu, is approximately $2 \times$ Delta $\mathrm{Tb}$ (K). TSM consistently under-predicts Delta $\mathrm{Tb}$ with respect to SPM/SSA across the whole range by a factor of about 2 . Implied model-dependent errors are of order $0.75 \mathrm{~K}$ in this case, corresponding to an SSS correction error of about 1.5 psu. This error can be compared with an SSS difference of $\sim 5$ psu observed across the Gulf Stream in 1999, using the PALS L-band radiometer [24].

\section{SUMMARY}

The dominant roughness influence on SSS retrieval comes from the shorter Bragg-scale components of the wind-wave spectrum $(1 \mathrm{~cm}-1 \mathrm{~m}$ at L-band). Since these waves, in particular, are poorly represented in traditional spectrum models, recent spectral model improvements have focused on better accounting for their effects using more sophisticated physical and empirical modeling [12] [18]. This seems preferable to simply doubling the spectral intensities to predict roughness emissivity influence. As the short wave components are modulated by the long waves, 
they are also influenced by swell [11], which future roughness correction models should also take into account.

We conclude that it is vital to choose the roughness correction model and input forcing function (wind speed, and/or spectrum) carefully to minimize this major error source for L-band SSS retrieval. Further enhancements to the $\mathrm{H}$ spectrum wind dependency are being investigated, and a rigorous FDTD reference model is currently under development as an aid in testing and enhancing candidate operational emissivity models. The results will be shared with the ESA SMOS and NASA Aquarius science teams.

\section{ACKNOWLEDGMENT}

This work was supported by the Office of Naval Research as part of the "Sea Surface Roughness Impacts on Microwave Sea Surface Salinity Measurements" research program under Program Element Number 0601153N. This is NRL contribution NRL/AB/7330-10-329.

\section{REFERENCES}

[1] D. M. Burrage, et al., "Optimizing performance of a microwave salinity mapper”, JAOT, 25 (5), pp 776-793, 2008.

[2] A. R. Camps, et al., "Sea Surface Emissivity at L-Band: Derived Dependence with incident and azimuth angles", Proc. of First Results Workshop on EuroSTARRS, WISE, LOSAC Campaigns, CESBIO, Toulouse, ESA SP-525, pp. 105-116, 2003.

[3] Camps, A., et al., "The WISE 2000 and 2001 field experiments in support of the SMOS Mission: Sea surface L-band brightness temperature observations and their application to sea surface salinity retrieval", IEEE, TGRS, 42, 804-823, 2004.

[4] C. Cox and W. H. Munk, "Statistics of the sea surface derived from sun glitter", J. Mar. Res, 13, 198-227, 1954.

[5] M. A. Donelan, et al., "Directional spectra of wind-generated waves”, Phil. Trans. Roy. Soc. Lond., A315,509-562, 1985.

[6] S. L. Durden, and J. F. Vesecky, "A physical radar crosssection model for a wind-driven sea with swell", IEEE, J. Oceanic Eng., 10, 445-451, 1985.

[7] T. Elfouhaily, et al., "A unified directional spectrum for long and short wind-driven waves", JGR, 102,C7, 15781-15796, 1997.

[8] J. Font, et al., "The determination of surface salinity with the European SMOS space mission”, TGRS, 42, 10, 2196-2205, 2004.

[9] C. Gabarro, et al. "Use of empirical sea surface emissivity models to determine sea surface salinity from an airborne L-band radiometer", Scientia Marina, June, 72 (2), pp 329-336, 2008.

[10] J. P. Hollinger, "Passive microwave measurements of sea surface roughness", IEEE Trans. Geos. Electron., 9, 165-169, 1971.
[11] P. A. Hwang, "Observations of swell influence on ocean surface roughness", JGR, 113, C12024 pp 1-14, doi:10.1029/ 2008JC005075, 2008.

[12] P. A. Hwang, "Wave number spectrum and mean-square slope of intermediate-scale ocean surface waves", JGR, 110, C10029, doi:10.1029/2005JC003002, 2005.

[13] P. A. Hwang, et al., "A study on the influence of ocean surface roughness spectral models on microwave brightness temperature computation", (In Revision, 2010).

[14] P. A. Hwang and W. J. Plant (2010), “An analysis of the effects of swell and roughness spectra on microwave backscatter from the ocean", JGR, 115, C04014, doi:10.1029/2009JC005558.

[15] V. G. Irisov, "Small-slope expansion for thermal and reflected radiation from a rough surface", Waves Random Media, 7, 1-10, 1997.

[16] J. T. Johnson and Min Zhang, "Theoretical Study of the Small Slope Approximation for Ocean Polarimetric Thermal Emission," TGRS, 37 (5), pp 2305-2316, 1999.

[17] L. Klein, and C. Swift, "An improved model for the dielectric constant of sea water at microwave frequencies," TAP 25 (1), pp 104-111, 1977.

[18] V. Kudryavtsev et al., "A semi-empirical model of the normalized radar cross section of the sea surface, 1. Background model," JGR, 108 (C3), 8054, doi:10.1029/2001JC001003, 2003.

[19] G. Lagerloef,"The Aquarius/SAC-D Mission.Oceanography," 21 (1), pp 68-81, 2008.

[20] T. Pérez, et al., "Airborne Remote Sensing of The Rio de la Plata Plume Using a Microwave Radiometer System", Sea Technology 47(9), 31-34, Sept., 2006.

[21] N. Reul, et al. "On the Use of Rigorous Microwave Interaction Models to Support Remote Sensing of Natural Surfaces”, IGARSS 2005, Proc. 3, pp 2195-2198, 2005.

[22] N. Reul, and B. Chapron, "SMOS- Salinity Data Processing Study: Improvements in emissivity models", IFREMER Tech. Rep. WP 1100, 130 pp., 2001.

[23] F. J. Wentz, (1976), Cox and Munk's sea surface slope variance, $J G R, 81,1607-1608$.

[24] W. J. Wilson, et al., "Ocean surface salinity remote sensing with the JPL Passive/Active L-/S-band (PALS) Microwave Instrument", IGARRS 2001, Sydney, Australia. 2, 937-939, 2001.

[25] S. H. Yueh, et al. (1994), Polarimetric passive remote sensing of ocean wind vectors, Radio Sci., 29, 799-814.

[26] S. Yueh, "Modeling of wind direction signals in polarimetric sea surface brightness temperatures", TGRS, 35, 6, 1400-1418, 1997. 